\title{
EGFR/HER1/HER2 Inhibitor PKI166
}

National Cancer Institute

\section{Source}

National Cancer Institute. EGFR/HER1/HER2 InhibitorPKI166. NCI Thesaurus. Code C1871.

A pyrrolo-pyrimidine epidermal growth factor receptor (EGFR) protein kinase inhibitor with anti-tumor activity. PKI-166 reversibly inhibits HER1 and HER2 tyrosine kinases, belong to the epidermal growth factor receptor family, thereby inhibiting tumor growth and metastasis. 